\title{
Meningioma extradural de oído medio: Reporte de un caso y revisión de la literatura
}

\section{Extradural meningioma of middle ear: Reporting a case and literature review}

\author{
Rodrigo Arregui $\mathbf{V}^{1}$, Romina Ovalle A' , Jorge Castillo A².
}

\begin{abstract}
RESUMEN
Los meningiomas son los tumores no gliales más comunes del sistema nervioso central constituyendo el 24\%-30\% de los tumores intracraneales y el $25 \%$ de los tumores de médula espinal. Se originan de células de la aracnoides y en general presentan un comportamiento benigno. Existe un subtipo llamado meningioma extracraneal primario o extradural que es poco frecuente y afecta principalmente el área de cabeza y cuello. Debido a su baja prevalencia y síntomas poco característicos son a menudo diagnosticados por fortuna, siendo la inmunohistoquímica fundamental. Se presenta el caso de una mujer adulta con una lesión tumoral en oído medio diagnosticada inicialmente mediante una biopsia incisional como un granuloma de colesterol. Luego del tratamiento quirúrgico y estudio de inmunohistoquímica se concluye el diagnóstico definitivo de meningioma extradural de oído medio. Se analiza la literatura al respecto y se discute sobre su epidemiología, clínica, estudio y manejo.
\end{abstract}

Palabras clave: Meningioma extracraneal primario, meningioma extradural, oído medio, inmunohistoquímica.

\begin{abstract}
Meningiomas are the most common non-glial tumors of the central nervous system constituting $24-30 \%$ of intracranial tumors and $25 \%$ of spinal cord tumors. They originate from arachnoid cells and generally exhibit benign behavior. The subtype primary extracranial meningioma or extradural meningioma that is uncommon and affects the head and neck area. Due to their low prevalence and uncharacteristic symptoms are often diagnosed by fortune, being the immunohistochemistry fundamental. We present the case of an adult woman with a tumor lesion in the middle ear initially diagnosed by an incisional biopsy such as a cholesterol granuloma. After the surgical treatment and immunohistochemical study, the definitive diagnosis of extradural meningioma of the middle ear is concluded. The literature on this subject is analyzed and its epidemiology, clinical practice, study and management are discussed.
\end{abstract}

Key words: Primary extracranial meningioma, extradural meningioma, middle ear, immunohistochemistry.

1 Médico Servicio de Otorrinolaringología, Hospital Barros Luco Trudeau.

2 Médico Servicio Anatomía Patológica, Hospital Barros Luco Trudeau. 


\section{INTRODUCCIÓN}

Los meningiomas son los tumores no gliales más comunes del sistema nervioso central constituyendo el $24 \%-30 \%$ de los tumores intracraneales y el $25 \%$ de los tumores de la médula espinal'1. Se originan de células de la aracnoides y en general presentan un comportamiento benigno². Según su ubicación se han descrito dos formas de meningiomas: los meningiomas intracraneales que son los más comunes y los meningiomas extracraneales que son más raros y que se subclasifican en meningiomas extracraneales primarios 0 extradurales y en meningiomas extracraneales secundarios ${ }^{1}$. Además, la Organización Mundial de la Salud los ha clasificado en tres grados según su nivel de malignidad: grado I o benigno, grado II 0 atípico y grado III 0 anaplásico. Independiente del tipo de meningioma, el tratamiento recomendado siempre ha sido la extirpación quirúrgica completa ${ }^{3}$. A continuación presentamos un caso de meningioma extradural de ubicación en oído medio y revisión de la literatura en el área de la otorrinolaringología.

\section{CASO CLÍNICO}

Mujer de 47 años, sin antecedentes médicos. Consulta en el Servicio de Otorrinolaringología del Hospital Barros Luco Trudeau (HBLT) por historia de un año de evolución de otorrea izquierda asociada a hipoacusia y acufeno ipsilateral. Al examen físico se encontró una perforación timpánica derecha y en el oído izquierdo destacaba un conducto auditivo externo (CAE) ocupado totalmente por una lesión polipoidea no pulsátil fácilmente sangrante que se biopsia en policlínico. Se solicita una audiometría que mostró una hipoacusia conductiva derecha (PTP vía aérea/ ósea: 48,3/20 dB) y una hipoacusia mixta izquierda (PTP vía aérea/ósea: 55/31,6 dB). Dentro del estudio imagenológico se solicitó una tomografía computarizada (TC) de peñasco con contraste que informó la presencia de un CAE izquierdo amplio ocupado por un contenido de densidad de partes blandas que llegaba a caja timpánica, antro y celdillas mastoideas, sin observar membrana timpánica ni tampoco destrucción ósea. La cadena osicular estaba englobada por este contenido pero sin signos de destrucción. La paciente llega a control ambulatorio con el resultado de la biopsia que informa hallazgos compatibles con granuloma de colesterol.

Con estos antecedentes se decide realizar una cirugía radical a demanda y extirpación de la lesión. En el intraoperatorio se encontró un CAE y una caja timpánica totalmente ocupados por una lesión tumoral de aspecto cerebriforme, blanda, no pulsátil, que se extirpa en su totalidad. La mucosa del oído medio se observaba inflamada y friable. Se realiza fresado de la pared lateral del ático observando lesión hasta ese nivel sin afección del tegmen. Se remueve el martillo y el yunque y se deja el estribo que se encuentra móvil. Se completa techo corrido tímpano-antro-mastoideo hasta encontrar celdillas mastoideas con mucosa sana. Se logra extirpar por completo la lesión y se deja sin cubrir la cavidad radical por el compromiso de la mucosa. El resultado de la biopsia diferida informa lesión tumoral compatible con meningioma de oído medio.

La paciente evoluciona satisfactoriamente en el posoperatorio y luego de un año de seguimiento no ha existido recidiva.

\section{DISCUSIÓN}

Los meningiomas extracraneales son tumores bastante raros constituyendo el $0,9 \%$ al $2 \%$ de todos los meningiomas ${ }^{1,4}$. En general son de comportamiento benigno ( $80 \%$ de los casos) y debido a su baja prevalencia y síntomas poco característicos son a menudo diagnosticados por fortuna.

Se subclasifican en dos tipos: meningioma extracraneal primario o extradural y meningioma extracraneal secundario. Los tumores primarios, también llamados no durales, extradurales o ectópicos, son aquellos que se aíslan totalmente como extracraneales sin tener asociación a ninguna masa intracraneal 5 . Por otra parte, los meningiomas secundarios que son más frecuentes que los extradurales, surgen como extensión de un tumor intracraneal a través de vías de extensión que se dirigen principalmente al territorio de cabeza y cuello (defectos quirúrgicos, forámenes en lámina cribosa y base de cráneo y vías óseas preformadas). 
Por lo tanto, una vez establecido el diagnóstico de meningioma, la presencia de un meningioma del tubo neural o la extensión de un meningioma extracraneal primario debe ser excluida. El 20\% de los tumores intracraneales presentan un componente extracraneal ${ }^{1,2}$.

La etiopatogenia de estos tumores reside en la migración de células aracnoideas a un lugar ectópico. Se han propuesto cuatro mecanismos posibles en la formación de meningiomas ectópicos: (a) extensión directa de una lesión intracraneal, (b) metástasis distante de un meningioma intracraneal, (c) origen desde las células aracnoideas presentes dentro de las vainas de los nervios craneales y (d) origen a partir de nidos de células embrionarias aracnoideas. En el caso de ubicaciones fuera de cabeza y cuello (pulmón, retroperitoneo, etc.) se cree que surgen de células aracnoideas presentes a lo largo de los nervios periféricos ${ }^{4}$.

La ubicación más frecuente de los meningiomas extradurales es el área de cabeza y cuello, siendo la cavidad nasal y las cavidades perinasales las más afectadas. También se han descrito casos en oído medio, hueso temporal, órbita, cuero cabelludo, glándula parótida, espacio submandibular y espacio parafaríngeo. Fuera del área de cabeza y cuello se han reportado casos en pulmón, mediastino, retroperitoneo y en pies ${ }^{5-8}$. Su presentación clínica es silenciosa hasta que alcanza cierto tamaño, comenzando a presentar síntomas según su localización.

En relación a los meningiomas extradurales de oído y hueso temporal representan $<1 \%$ de todos los meningiomas. Thompson y cols ${ }^{9}$, en el año 2002, presentaron 36 casos de meningiomas extradurales de oído y hueso temporal encontrados en el Registry of the Armed Forces Institute of Pathology (AFIP) entre los años 1970 y 1996, constituyendo la serie con mayor número de casos reportados. La edad de presentación varió entre los 10-80 años, siendo 24 casos mujeres y 12 casos hombres. Dentro de la sintomatología se describió hipoacusia, otitis, dolor y vértigo. Los síntomas se presentaron durante una media de 24,6 meses antes del diagnóstico. En cuanto a su ubicación 25 casos se encontraron en oído medio, 4 casos en conducto auditivo externo y 7 casos con compromiso tanto de oído medio como de hueso temporal.
Respecto al diagnóstico de los meningiomas la inmunohistoquímica es fundamental. Sin embargo, no hay diferencias histológicas que permitan diferenciar un tipo intracraneal de uno extracraneal por lo que su ubicación permite hacer este diagnóstico diferencial'1. A la histología los meningiomas se caracterizan por presentar nidos sólidos de células meningoteliales dispuestas en hojas 0 verticilos en un fondo que a menudo es fibroadiposo y pueden presentar cuerpos de psamomma. Se reconocen cuatro patrones microscópicos de meningiomas: el tipo sincicial, que consiste en una lámina uniforme de células poligonales; una forma transitoria 0 psamommatosa con presencia de células fusiformes y cuerpos de psammoma; una forma fibrosa con celularidad reducida y aumento del contenido de colágeno; y el tipo angioblástico con alta celularidad y con una forma sincicial 0 de transición adyacente ${ }^{2}$. La mayoría de los meningiomas extracraneales son del tipo sincicial 0 de transición². En la inmunohistoquímica estos tipos de tumores tienen una fuerte tendencia a ser positivos para vimentina y para el Antígeno de Membrana Epitelial (EMA) mientras que frente a proteína S-100 y queratina (KER) se han descrito resultados variables desde positividad focal hasta ausencia de reacción ${ }^{1,4,5}$. Expresión de E-cadherina también se ha descrito como característico en meningiomas intracraneales ${ }^{5}$. En nuestro caso clínico se realizó análisis de inmunohistoquímica para VIM, EMA, S-100 y KER resultando la muestra positiva para VIM y EMA, y negativa para S-100 y KER (Figura 1). El diagnóstico diferencial debe realizarse con paragangliomas, schwannomas, adenomas, carcinomas y melanomas ${ }^{5,9}$.

En relación al tratamiento, independiente del tipo de meningioma, siempre debe intentarse la extirpación quirúrgica completa. Las recurrencias generalmente se desarrollan en el mismo sitio que la lesión primaria y representan probablemente la enfermedad residual en lugar de un tumor recurrente. Las características clínicas y radiológicas de estos tumores no pueden predecir la naturaleza de estas lesiones. Por lo tanto, el examen histopatológico es necesario para el diagnóstico diferencial y para establecer el comportamiento del meningioma ${ }^{2,6,9}$. 


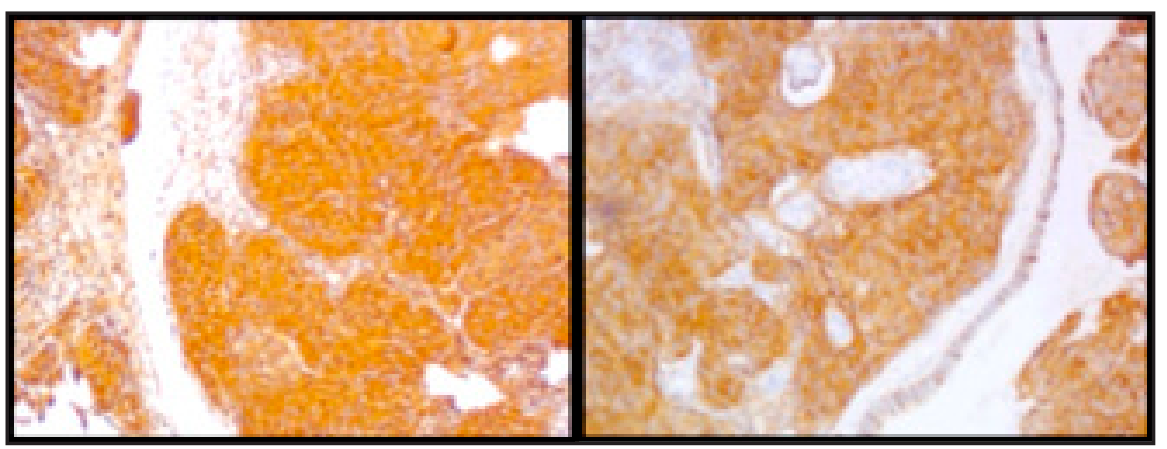

Figura 1. Corte histológico. Inmunohistoquímica positiva para vimentina (a la izquierda) y para EMA (a la derecha).

\section{CONCLUSIÓN}

Los meningiomas extracraneales primarios 0 extradurales son raros, pero siempre deben considerarse como diagnóstico diferencial de masas en la región de cabeza y cuello. El

\section{BIBLIOGRAFÍA}

1. Albsoul N, Rawashdeh B, Albsoul A, et al. $A$ rare case of extracranial meningioma in parapharyngeal space presented as a neck mass. International Journal of Surgery Case Reports 2015; 11: 40-3.

2. Possanzini P, Pipolo C, Romagnolis, et al. Primary extra-cranial meningioma of head and neck: clinical, histopathological and immunohistochemical study of three cases. Acta Otorhinolaryngol Ital 2012; 32: 336-8.

3. Serry P, Rombaux PH, Ledeghen S, et al. Extracranial sinosal tract meningioma: a case report. Acta Otorhinolaryngol Bel 2004; 58: 151-5.

4. Deshmukh S, Rokade V, Pathak G, Nemade S, AshtuRkar A. Primary extra-cranial meningioma in the right submandibular region of an 18 year old woman: a case report. Journal of Medical Case Reports 2011; 5: 271-4. diagnóstico definitivo lo establece la histología, fundamentalmente la inmunohistoquímica, y el tratamiento de elección es la escisión quirúrgica completa. Presentan buen pronóstico luego de la resección quirúrgica, con bajas tasas de recurrencia.

5. Tomaru U, Hasegawa T, Hasegawa F, Kito M, Hirose, Shimoda T. Primary Extracranial Meningioma of the Foot: A case report. Jpn J Clin Oncol 2000; 30: 313-7.

6. Drlicek M, Grisold W, Lorber J, Hackl H, WuKETICH S, JILLINGER K. Pulmonary Meningioma. Inmunohistochemical and ultrastructural features. Am J Surg Pathol 1991; 15: 455-9.

7. Wilson AJ, Ratliff JL, Lagios MD, Aguilar MJ. Mediastinal Meningioma. Am J Surg Pathol 1979; 3: 557-62.

8. Huszar M, Fanburg JC, Dickersin GR, Kirshner JJ, Rosenberg AE. Retroperitoneal malignant meningioma. A light microscopic, inmunohistochemical and ultrastructural study. Am J Surg Pathol 1996; 20: 492-9.

9. Thompson LDR, Bouffard JP, Sandberg GD, Mena H. Primary Ear and Temporal Bone Meningiomas: A Clinicopathologic Study of 36 Cases with a Review of the Literature. Mod Pathol 2003; 16: 236-45.

Dirección: Rodrigo Arregui V. 